\title{
Reprogrammed cells dissect ape retrotransposition
}

both
APOBEC3B
and PIWIL2
expression
levels are
causally
linked to this
differential

LINE- 1 activity
Our ability to address the important question of how differences between humans and other primates have evolved is limited by the study systems that are available for doing so. A new study uses induced pluripotent stem cells (iPSCs) of primates to identify differential regulation of long interspersed element 1 (LINE-1) retrotransposons between humans and non-human primates (NHPs).

The characterization of biological processes in primates is hindered by the limited availability of primary cells and tissues, particularly those of embryonic origin. To circumvent this limitation Marchetto et al. used pluripotency factor genes to reprogramme fibroblasts from two chimpanzees and two bonobos into iPSCs. Comparisons with human embryonic stem cells and equivalently derived human iPSCs revealed similar gene expression profiles and in vitro differentiation properties across all these pluripotent cell lines.

Among the few differentially expressed genes, PIWI-like RNAmediated gene silencing 2 (PIWIL2) and the cytidine deaminase gene
$A P O B E C 3 B$ were upregulated in human iPSCs relative to NHP iPSCs. Both of these genes have known links to silencing LINE-1 elements in mammalian germlines to limit retrotransposition. By transfecting a plasmid reporter of LINE-1 activity into both human and NHP iPSCs, the authors indeed identified greater LINE-1 activity in NHP iPSCs. Furthermore, by manipulating the expression of PIWIL2 and APOBEC $3 B$ (through overexpression or knockdown) in these iPSCs, they showed that both $A P O B E C 3 B$ and PIWIL2 expression levels are causally linked to this differential LINE-1 activity between species. Although the mechanism by which $A P O B E C 3 B$ limits LINE-1 activity remains unclear, LINE-1complementary PIWI-interacting RNAs (piRNAs) were found in human iPSCs, which is consistent with a piRNA-mediated role for PIWIL2 in controlling LINE-1 activity in human iPSCs.

Finally, the authors examined endogenous LINE-1 activity higher levels of LINE-1 transcripts were found in NHP iPSCs than in human iPSCs, and genome sequence analyses revealed more chimpanzeespecific than human-specific LINE-1 insertions. Cumulatively, the data indicate a higher activity and mobility of LINE-1 elements in NHPs relative to humans.

It will be interesting to determine the extent to which differential activity of LINE-1 or other transposable elements contributes to germline mutation rates, inter-individual variability and adaptive potential among primates, and to explore how iPSCs from various species can facilitate future biological investigations.

Darren J. Burgess

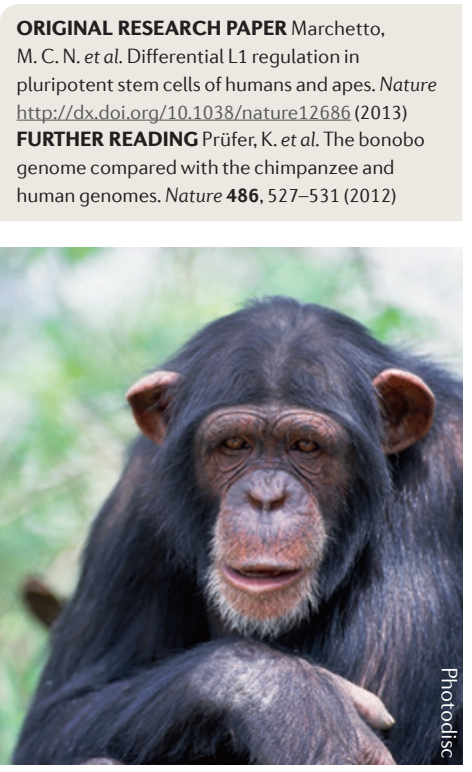

